\title{
Measurements of tangential momentum accommodation coefficient for various gases in plane microchannel
}

\author{
I. A. Graur, ${ }^{\text {a) }}$ P. Perrier, W. Ghozlani, and J. G. Méolans \\ Département de Mécanique Energétique-UMR CNRS 6595, Université de Provence-Ecole Polytechnique \\ Universitaire de Marseille, 5 rue Enrico Fermi, 13453 Marseille Cedex 13, France
}

(Received 3 March 2009; accepted 18 September 2009; published online 30 October 2009)

\begin{abstract}
Mass flow rate measurements in a single silicon microchannel were carried out for various gases in isothermal steady flows. The results obtained from hydrodynamic to near free molecular regime by using a powerful experimental platform allowed us to deduce interesting information, notably about the reflection/accommodation process at the wall. In the $0-0.3$ Knudsen range, a continuum analytic approach was derived from the NS equations, associated with first or second order slip boundary conditions. Identifying the experimental mass flow rate curves to the theoretical ones the tangential momentum accommodation coefficient (TMAC) of various gases was extracted. Over the full Knudsen range [0-30] the experimental results were compared with theoretical values calculated from the kinetic approaches: using variable accommodation coefficient values as fitting parameter, the theoretical curves were fitted to the experimental ones. Whatever the Knudsen range and whatever the theoretical approach, the TMAC values are found decreasing when the molecular weights of the gas increase (as long as the different gases are compared using the same approach). Moreover, the values of the various accommodation coefficients are rather close to one another but sufficiently smaller than unity indicating that the full accommodation modeling is not satisfactory to describe the gas/wall interaction. (C) 2009 American Institute of Physics. [doi:10.1063/1.3253696]
\end{abstract}

\section{INTRODUCTION}

Since the early 1980 s and the beginning of the developments of the microelectromechanical systems (MEMS), a lot of microdevices has been designed to study experimentally gas microflows, in the channel geometry. ${ }^{1-3}$ Nevertheless, the experiments concerning a single conduit and a pressure domain similar to those investigated here $\left(6.04 \times 0^{1}-1.098\right.$ $\left.\times 10^{5} \mathrm{~Pa}\right)$ were rare; mainly because such experiments correspond to mass flow rates lower than $10^{-10} \mathrm{~kg} / \mathrm{s}$, which are difficult to measure.

The purpose of the present study is twofold. The main part of the work is devoted to the comparison of the properties of various gases flowing in a plane channel geometry. Then a second aim is to compare the result obtained in the channel to those previously found in microtubes. Thus the results obtained here with argon and nitrogen were joined to those previously obtained for helium in the same plane channel (the details on the channel material and fabrication technique are given in Sec. II) and compared together. In the global range of Knudsen numbers investigated here, a special treatment was devoted to the hydrodynamic and slip regimes located in the [0-0.3] Knudsen range. In this range it is theoretically justified to use the continuum Navier-Stokes equations. Moreover the channel geometry (height to length ratio $H / L \sim 10^{-3}$, small compared to unity) allowed a small perturbation treatment leading to approximated simple equations. Thus, associated with first or second order slip boundary conditions, depending on the gas, these equations led to an analytic expression of the mass flow rate. This mass flow

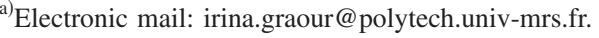

rate depends on the velocity slip coefficient and then on the accommodation coefficient. The modus operandi of validation of the first or second order boundary conditions was detailed and justified elsewhere, ${ }^{4}$ see also Sec. III. Thus, identifying the experimental mass flow rate curves to the theoretical ones the tangential momentum accommodation coefficient (TMAC) of various gases were extracted.

On the other hand, over all the Knudsen range [0-0.3] the experimental results were compared with theoretical values calculated from the kinetic approaches. Using variable accommodation coefficient values as fitting parameter, the theoretical curves were compared to the experimental ones and the TMAC value giving the best fit was retained for each gas. Globally an overall agreement appears on the large Knudsen range investigated, involving some constant features, notably:

- A same trend according to the gases and regarding as well the accommodation coefficient as the second order effect was found in slip regime,

- A non-negligible number of the particle reflection on the wall occurs in the specular way, where the molecules reflect with the temperature of the incoming molecules and with a velocity symmetric to that of the incoming molecules with respect to the normal at the wall.

These results call into question various approaches where the full accommodation at the wall is assumed. 


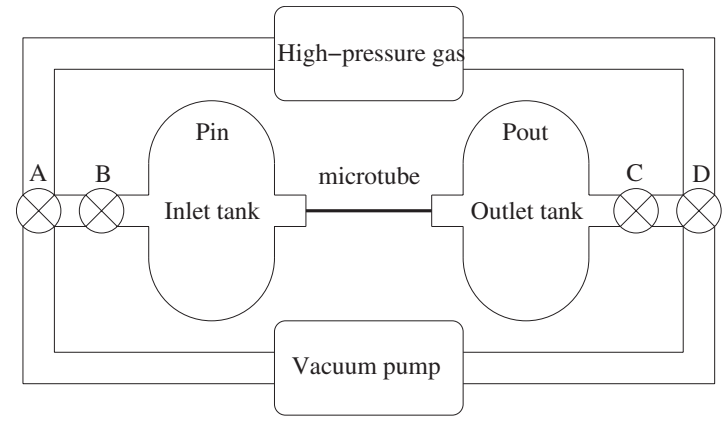

FIG. 1. Mass flow experiment diagram. The valves A, B, C, and D are used to impose and to adjust the pressure in the inlet and outlet tanks.

\section{MEASUREMENTS}

\section{A. Experimental setup}

The experimental method used in the present work was denoted "constant-volume technique." This method requires very large tank volumes, much larger than the volume of the microchannel. Large tank sizes guarantee that the microflow parameters are independent of time; although detectable, the mass variations occurring in the tanks during the experiments do not call into question the steady assumption. Thus, we also have to set a limit for the maximal suitable variation of the pressure in the second tank, according to the inlet and outlet conditions. These constraints are taken into account in the experimental setup shown in Fig. 1. The gas flows through a silicon microchannel fixed between two tanks in which the pressures remain very close to constant values $P_{\text {in }}$ and $P_{\text {out }}$, respectively. The pressure variation in the second tank due to the gas flow through the microchannel is set at $\pm 1 \%$ of the tank pressure averaged over the experiment duration. Consequently, the relative pressure variation in the first tank remains close to $\pm(0.33 \%-0.5 \%)$ depending on the pressure ratio between the tanks. This pressure variation range corresponds to a required experiment duration $\tau$ ranging from about a few seconds for the highest mass flow rate measured $\left(10^{-8} \mathrm{~kg} / \mathrm{s}\right)$ to about $120 \mathrm{~s}$ for the lowest $\left(10^{-13} \mathrm{~kg} / \mathrm{s}\right)$. The more detailed description of the experimental set up may be found in Ref. 4. The estimation of the leakage may be also found in Ref. 4.

\section{B. Microchannel characteristics}

The microchannel is microfabricated using standard techniques in clean room facilities of the Technology platform LAAS and LETI. The size of the silicon wafer is 3 in. (diameter) and $350 \mu \mathrm{m}$ (thickness). The channel is obtained by anisotropic etching limited by the crystal planes (111 and $100)$ and after the direct bounding $(\mathrm{Si} \mathrm{Si})$ is carried out. The dimensions (height $H$ equals $9.38 \pm 0.2 \mu \mathrm{m}$ and width $W$ equals $492 \pm 1 \mu \mathrm{m})$ are measured for the entrance and output sections by the microscope electron beam (MEB) after the bonding, the channel length $L$ equals $9.39 \pm 0.1 \mathrm{~mm}$. Like in Ref. 4 the ratio between the transversal dimension and the length of the conduit is close to $10^{-3}$. The wall roughness is only estimated after bonding using the electron microscope and this estimation is limited by the resolution of the MEB, so the roughness of the channel walls is estimated to be smaller than $20 \mathrm{~nm}$.

\section{Mass flow rate measurements and nonisothermal effects}

The technique of the mass flow rate measurements consists of determining in the outlet tank a small pressure change due to the mass flowing from the microchannel. The ideal gas low in the outlet tank reads

$$
P_{\text {out }} V=m \mathcal{R} T,
$$

where $V$ represents the outlet tank volume which remains constant during the experiment, and $\mathcal{R}$ is the specific gas constant. $P_{\text {out }}, T$, and $m$ are, respectively, the pressure, temperature, and mass of the gas in the outlet tank at any time $t$ of the experiment time length $\tau$. As it is shown in Ref. 4, when the relative temperature variation is negligible compared to the relative pressure variation $\delta P_{\text {out }} / P_{\text {out }}$ during the experiment duration $\tau$, the quantity $d m / \tau$ may be identified as the mass flow rate $\dot{M}$, which reads

$$
\dot{M}=\frac{V}{R T} \frac{\delta P_{\text {out }}}{\tau} .
$$

The experiments are performed within a narrow temperature range, excluding any heat source in the environment. During each experiment, the temperature $T$ is not maintained but controlled to be sufficiently constant to justify the isothermal assumption.

\section{Pressure measurements}

As in Ref. 4 the pressure variation measured in the second tank was smaller than or equal to $\pm 1 \%$ of the tank pressure, allowing a steady isothermal treatment. The pressure measurements are carried out using simultaneously two detectors chosen according to the pressure range (see Refs. 4 and 5 for more details). One is located in the first tank upstream from the microchannel and the other in the second tank downstream from the microchannel. The errors in pressure measurements in each tank depend on the characteristics of the pressure detectors; the details are given in Refs. 4 and 5 . Thus, in the pressure range observed during the experiments, the errors on the measurement of the outlet pressures may be estimated smaller than $0.5 \%$.

Each experiment is carried out with a constant pressure ratio $\mathcal{P}$ between the tanks with pure gases $(99.999 \%$, impurity less than $6 \mathrm{ppm})$. The real pressure ratios $\mathcal{P}_{E}$ maintained in each experiment lie within narrow bands centered around pressure ratio $\mathcal{P}_{C}$ close to 3 for helium flow and close to 2 for argon and nitrogen flows. The properties of these three series are summarized in Table I. The variation of the Knudsen number (or of the rarefaction parameter) is carried out using simultaneous changes in $P_{\text {in }}$ and $P_{\text {out }}$ values keeping their ratio close to the ratio fixed for a series of measurements.

Since the effects of the temperature variation are negligible, we may consider the flow through the microchannel as 
TABLE I. Experimental range of quantities: $\mathcal{P}$ represents the desired ratio $P_{\text {in }} / P_{\text {out }}$, whereas $\mathcal{P}_{E}$ is the real ratio for each measurement, and $\mathcal{P}_{C}$ is the mean experimental value of $\mathcal{P}_{E} . \Delta Q / Q$ is the relative uncertainty of the quantities $Q$.

\begin{tabular}{|c|c|c|c|c|c|c|c|}
\hline \multirow[b]{3}{*}{ Quantity } & \multirow{3}{*}{$\underset{(\%)}{\Delta Q / Q}$} & \multicolumn{6}{|c|}{ Gas } \\
\hline & & \multicolumn{2}{|r|}{$\mathrm{Ar}$} & \multicolumn{2}{|c|}{$\mathrm{N}_{2}$} & \multicolumn{2}{|c|}{$\mathrm{He}$} \\
\hline & & Min. & Max. & Min. & Max. & Min. & Max. \\
\hline Mass flow rate $(\mathrm{kg} / \mathrm{s})$ & 4.5 & $3.24 \times 10^{-12}$ & $1.09 \times 10^{-8}$ & $4.61 \times 10^{-13}$ & $1.34 \times 10^{-8}$ & $8.16 \times 10^{-13}$ & $1.51 \times 10^{-9}$ \\
\hline Inlet pressure $(\mathrm{Pa})$ & 0.2 & 267.31 & 100417.0 & 30.498 & 119727.0 & 121.24 & 96665.0 \\
\hline Outlet pressure $(\mathrm{Pa})$ & 0.2 & 122.03 & 49876.0 & 14.61 & 60129.0 & 40.35 & 32654.0 \\
\hline Average Knudsen number $K n_{m}$ & 2.5 & 0.00984 & 3.76 & 0.00765 & 30.5 & 0.0319 & 25.7 \\
\hline Experiment $\mathcal{P}_{E}$ & & 1.96 & 2.24 & 1.86 & 2.19 & 2.90 & 3.06 \\
\hline Centered $\mathcal{P}_{C}$ & & \multicolumn{2}{|r|}{2.02} & \multicolumn{2}{|c|}{2.02} & \multicolumn{2}{|c|}{2.99} \\
\hline Temperature $(\mathrm{K})$ & 0.2 & 292.9 & 295.9 & 291.6 & 293.8 & 294.2 & 297.7 \\
\hline
\end{tabular}

a steady flow occurring between two tanks maintained at pressures $P_{\text {in }}$ and $P_{\text {out }}$, practically constant, respectively, with variations of the order of $\pm 1 \%$ in the outlet tank and of the order of $\pm(0.33 \%-0.5 \%)$ in the inlet tank (see Sec. II A). These variations, smaller than the experimental error, are consistent with the steady assumption. Moreover, the isothermal mass flow rate may be expressed in form (2). To determine this mass flow rate we will use the registered data for pressures $P_{i}$ at time instants $t_{i}$. The stationary flow conditions physically justify a pressure rise interpolation by means of a linear function of time using a simple least-squares fit:

$$
P_{f}(t)=a t+b, \quad a=\frac{\delta P_{\mathrm{out}}}{\tau} .
$$

As it was explained in Ref. 5, the error on the coefficient $a$ is of the order of $\pm 0.5 \%$, highly overestimating this value.

Since the leaks are estimated as totally negligible, ${ }^{4}$ we do not integrate them in the uncertainty on the mass flow rate. Therefore, we obtain a full uncertainty on $\Delta \dot{M} / \dot{M}$ smaller than $\pm 4.5 \%$ ( $2 \%$ due to the temperature, $2 \%$ due to the volume, $0.5 \%$ due to the slope of pressure; see Table I and Ref. 4 for details).

\section{BACKGROUND THEORY}

\section{A. Hydrodynamic and slip regime}

For many years, pressure-driven slip flows within ducts or channels received considerable attention. Many formulations of analytical and semianalytical solutions have been presented. ${ }^{6,7}$ The analytical models derived from the NavierStokes equations or from other continuum equation systems require the use of the velocity slip boundary conditions. Despite numerous previous studies, the choice of the appropriate boundary conditions (first or second order) and the upper boundary of continuum equation validity remained open questions. As we said in the Introduction, we recently started methodically to clarify this questions. ${ }^{4-8}$ Thus we proved the validity of using second order boundary condition on the Knudsen number range $0.003-0.3$ in a microtube. Therefore the flow analysis was made hereafter a priori with the Navier-Stokes equations using second order slip boundary conditions at the wall; but the pertinence of this approach (compared to the first order approach) will be verified in next section. Then the slip velocity reads

$$
u_{s}= \pm A_{1} \lambda\left(\frac{\partial u}{\partial y}\right)_{w}-A_{2} \lambda^{2}\left(\frac{\partial^{2} u}{\partial y^{2}}\right)_{w},
$$

where $\lambda$ is the mean free path of the molecules $\lambda=k_{\lambda}(\mu / P) \sqrt{2 R T}$ and $\mu$ is the viscosity coefficient. The coefficient $k_{\lambda}$ used in the expression of the mean free path depends on the intermolecular interaction model. For the hard sphere model, ${ }^{9} k_{\lambda}$ is equal to $\sqrt{\pi} / 2$. The coefficients $A_{1}$ and $A_{2}$ in Eq. (4) may be presented in the form

$$
A_{1}=\frac{\sigma_{p}}{k_{\lambda}}, \quad A_{2}=\frac{\sigma_{2 p}}{k_{\lambda}^{2}},
$$

where $\sigma_{p}$ and $\sigma_{2 p}$ are the first and second velocity slip coefficients. Then the nondimensional mass flow rate $S$ through the microchannel obtained from Navier-Stokes equations using the second order velocity slip condition ${ }^{5}$ reads

$$
S=\dot{M} / \frac{H^{3} w \Delta P P_{m}}{12 \mu R T L}=1+6 A_{1} K n_{m}+6 A_{2} \frac{\mathcal{P}+1}{\mathcal{P}-1} \ln \mathcal{P} K n_{m}^{2},
$$

where $w$ is the channel width, $\Delta P=P_{\text {in }}-P_{\text {out }}, \mathcal{P}=P_{\text {in }} / P_{\text {out }}$, and where $K n_{m}$ is the mean Knudsen number, based on the mean pressure $P_{m}=0.5\left(P_{\text {in }}+P_{\text {out }}\right)$. Expression (6) may be rewritten in the more compact form:

$$
S^{\text {theor }}=1+A^{\text {theor }} K n_{m}+B^{\text {theor }} K n_{m}^{2} .
$$

The analytical expressions of the mass flow rate (6) and (7) were used for the calculation; they were compared with the fitting curve of the appropriate measured values. From this comparison, we deduce the "experimental" velocity slip coefficient, and then, the experimental accommodation coefficient $\alpha$ [Eq. (13)], thereafter identified as the TMAC.

As it is well known the coefficient $\alpha$ appears first in the kinetic boundary condition at the wall. In the specular/ diffuse Maxwell law employed in this work, $\alpha$ denotes the first the part of molecules reflected following the diffuse way (i.e., as being in local equilibrium with the wall or "accommodated with the wall"). Then, it is shown ${ }^{10}$ that, for various kinetic quantities $Q$, this coefficient also equals a character- 
istic ratio concerning the flux of each of these quantities transferred to (or from) the wall. For the flux of each kinetic quantity $Q, \alpha$ equals the ratio of its actual transferred value to the maximum possible transfer. However this maximum transfer is reached when the reflected molecules are "fully accommodated to the wall." Therefore $\alpha$ is called "accommodation coefficient of $Q . "$

Theoretically, the accommodation coefficient present in the Maxwell law may be related to various flux ratios: $\alpha$ equals the ratio concerning the tangential momentum component as well as that of the normal momentum component or that of the total energy. But practically the correct meaning of $\alpha$ depends on the physical conditions and on the experimental technique used for the measurements.

In isothermal flows where neither thermal gradient nor thermal jump at the wall are present, the coefficient acting on the measured quantities (as the mass flow rate) is usually identified as the TMAC. More especially, in microchannels for isothermal slip regime, the mass flow rate is explicitly related to $\alpha$ via the slip coefficient $\sigma_{p}$. This usual $\sigma_{p}$ expression (as $\alpha$ function) is calculated notably using a momentum balancing of Maxwell, ${ }^{11}$ where $\alpha$ is identified as the TMAC.

\section{B. Transitional and near free molecular regimes}

The most complicated domain for modeling is the transition flow regime, where the Boltzmann equation should be solved. A review of the main results obtained in this field may be found in Ref. 12. We detailed only the results which were the most useful for the analysis of our experimental measurements. We start with the modeling of the flow between two parallel plates. The expression of a volume flow rate between two parallel plates, for a large Knudsen number range, was first obtained by Ref. 13 solving the BGK kinetic equation, which governs the distribution function, by using the discrete ordinate method and assuming a diffuse reflection of the molecules from the wall. The BGK model, again, then associated with a diffuse-specular reflection on the solid surface, was also considered by Ref. 14. Moreover the linearized Boltzmann equation for the flow between two parallel plates was solved by Refs. 15 and 7 for hard-sphere molecules using a diffuse reflection as boundary condition. The difference between the solution of the BGK equation and that of the Boltzmann equation is about only $2 \% .^{12}$ The finite width dimension of the rectangular channel section was taken into account by Ref. 16 when solving numerically the BGK kinetic equation using a diffuse scattering. In this case of rectangular channels with arbitrary height to width ratios, two modelings of the collision integral, the BGK model ${ }^{17}$ and the $\mathrm{S}$ model, ${ }^{18}$ both assuming a complete accommodation of the molecules on the wall, were compared in the isothermal case; the difference between the mass flow rates obtained with these two models is less than $1 \% .{ }^{12}$ Moreover, as seen above, the difference between the solution of the linearized Boltzmann equation and those of the two kinetic equation models is also very small; thus, in order to compare the theoretical results with our measurements, we used below only the results given by the solution of the BGK model,
Refs. 17 and 14, which take, respectively, into account the finite width of the channel or the incomplete accommodation at the wall.

In all the approaches presented above the same basic assumption is made: the local pressure gradient, defined as follows, is small:

$$
\nu=\frac{H}{P} \frac{d P}{d x} \ll 1 .
$$

Then according to many authors, quoted above, this assumption allows the linearization of the kinetic equation and that of the boundary condition around the equilibrium state. The dimensionless mass flow rate reads

$$
Q=-\frac{\sqrt{2 R T}}{H w P \nu} \dot{M},
$$

when the local pressure gradient $d P / d x$ does not vary too much along the channel, expression (9) does not either. Moreover, in such conditions, the dimensionless flow rate is roughly independent of the local pressure gradient characterizing the flow but depends mainly on its rarefaction parameter: $\delta=(\sqrt{\pi} / 2)(H / \lambda)\left(\delta \sim K n^{-1}\right)$. This nondimensional mass flow rate (9) considered as a function of the local rarefaction parameter $\delta$ was calculated by many authors. ${ }^{14,16,15,17,18}$ In fact in all these theoretical papers, the rarefaction parameter $\delta$ was assumed to be practically constant along the channel. This assumption seems to be much more restrictive than expression (8). But, in our experimental measurements, the pressure variations along the channel were important (the ratio between the pressures in the inlet and outlet tanks may be equal to 3), and so the pressure ratios were high compared to 1 and the rarefaction parameter is no longer constant along the channel. However, some authors ${ }^{12}$ consider that since for a channel with $H / L \ll 1$ the local pressure gradient defined as in relation (8) is always small at any pressure ratio, it is possible to apply this theoretical approach in the case of high pressure ratios provided that the changes in the rarefaction parameter due to the pressure changes are taken into account.

In order to compare our experimental results with existing theoretical models we adopted these authors' point of view and we implemented this proposed approach. Moreover, it would be difficult to compare locally the measured and the calculated mass flow rates using the dimensionless form (9), since the local pressure and local rarefaction parameter are unknown (we do not measure the pressure distribution along the channel). Thus, following Ref. 12, this issue is overcome integrating the two members of relation (9) along the channel and using the property of the mass flow rate $(\dot{M})$ conservation; we deduce the following relations:

$$
\begin{aligned}
& G=\frac{L \sqrt{2 R T}}{H^{2} w\left(P_{\text {in }}-P_{\text {out }}\right)} \dot{M}, \quad \text { with } \\
& G\left(\delta_{\text {in }}, \delta_{\text {out }}\right)=\frac{1}{\delta_{\text {out }}-\delta_{\text {in }}} \int_{\delta_{\text {in }}}^{\delta_{\text {out }}} Q(\delta) d \delta .
\end{aligned}
$$

As noted in Ref. 17, we remark that $G$, which is the mean value of $Q$ along the channel, does not depend any longer on 
TABLE II. The pertinent experimental coefficients $A^{\exp }$ and $B^{\exp }$ are obtained from the first order or second order polynomial fitting. The first order helium results are derived from Ref. 5. The values in bold characters indicate the retained values for each gas.

\begin{tabular}{lcccccc}
\hline \hline Gas & Order & $A^{\text {exp }}$ & $B^{\text {exp }}$ & $s_{r}$ & $r^{2}$ & $\begin{array}{c}E_{s} \\
(\%)\end{array}$ \\
\hline $\mathrm{He}$ & First & $\mathbf{7 . 8 0 6} \pm \mathbf{0 . 0 5 1}$ & 0 & 0.0252 & 0.998 & 1.38 \\
& Second & $7.721 \pm 0.154$ & $0.456 \pm 0.798$ & 0.0258 & 0.998 & 1.36 \\
$\mathrm{~N}_{2}$ & First & $8.485 \pm 0.072$ & 0 & 0.0347 & 0.997 & 2.21 \\
& Second & $\mathbf{7 . 4 7 3} \pm \mathbf{0 . 0 6 6}$ & $\mathbf{5 . 2 3 3} \pm \mathbf{0 . 3 2 6}$ & 0.010 & 0.999 & 0.621 \\
$\mathrm{Ar}$ & First & $9.173 \pm 0.147$ & 0 & 0.106 & 0.984 & 5.41 \\
& Second & $\mathbf{8 . 1 5 6} \pm \mathbf{0 . 4 3 1}$ & $\mathbf{4 . 7 7 2} \pm \mathbf{1 . 9 1 9}$ & 0.097 & 0.987 & 4.87 \\
\hline \hline
\end{tabular}

the local pressure gradient, but only on its mean value. Relations (10) written above may be used to calculate numerically the reduced mass flow rate $G\left(\delta_{\text {in }}, \delta_{\text {out }}\right)$ from the table of $Q(\delta)$ given in Refs. 17 and 19, where this nondimensional mass flow rate $Q$ has been obtained using the BGK kinetic equation associated with a diffuse-specular scattering.

It is to note that the correct use of relation (10) requires that $Q(\delta)$ depends only on $\delta$, excluding a local dependence on the pressure gradient. However relation (10) defines a quantity $Q$ formally independent of the pressure gradient only when we can use the slip regime expression of $\dot{M}$. In the other regimes, the property is exact only if the pressure gradients are constant (i.e., for a linear pressure profile along the conduit). This approximation could be less and less good for example for high variation of the pressure gradient occurring in transitional regime.

Let us now estimate the possible numerical and theoretical errors concerning the adopted approach. From the numerical point of view the tables of the mass flow rate values are calculated from the solution of the linearized BGK kinetic equation, which is an approximation of the Boltzmann equation. The mass flow rate $Q(\delta)$ is obtained from the solution of BGK equation, following Ref. 17, with the accuracy of $0.1 \%$. Then, in order to calculate the reduced mass flow rate $G\left(\delta_{\text {in }}, \delta_{\text {out }}\right)$ the tabulated values of $Q(\delta)$ are interpolated and then integrated using the simple trapezoidal rules, so this procedure may decrease the accuracy until $1 \%$. Therefore, this implemented technique does not induce a more important error than $1 \%$, which represents a reasonable accuracy.

But this evaluation does not include the departure from the exact theoretical value coming from the model assumptions, not exactly fulfilled anywhere. We can give a rough estimation of this theoretical "error" as follows. In the slip regime, with conditions similar to our experimental conditions, the difference between the actual pressure profile (parabolic) and the linear profile varies from zero to about $3 \%$ along the conduit axis. ${ }^{20}$ It is to note that this evaluation overestimates the difference occurring in the transitional regime, where $Q$ is still dependent on the pressure gradient but where the pressure profile is smoothed when the Knudsen number increases. Using an analytic slip regime expression we can deduce the order of magnitude of the resulting departure on the mass flow rate, namely, $6 \%$. Now using an algebraic average along the channel, we can evaluate the result- ing departure upon $G$ as about $5 \%$. As previously said, maximizing the nonlinearity of the pressure profile, we overestimated the resulting approximation on $G$, which probably remains generally under 5\%. Nevertheless we cannot exclude, in any case, an approximation overshooting the experimental uncertainty. This question remains an open question and our measurements give also a part of the response.

\section{RESULTS AND DISCUSSION}

Two gas flows $\left(\mathrm{N}_{2}, \mathrm{Ar}\right)$ were studied for the different flow regimes: from the hydrodynamic regime for which the mean Knudsen number is equal to 0.007 to the near free molecular regime corresponding to a mean Knudsen number equal to 30. Each experiment was carried out for a constant pressure ratio, $\mathcal{P}=2$, between the tanks. The maximum Knudsen number was reached for $\mathrm{N}_{2}$. The total investigated Knudsen number range was much too wide $(0.007-30)$ to be globally analyzed in the frame of a first or second order treatment using the continuum approach. Therefore we split the total Knudsen number range in several parts and we considered a first part of this range below a maximum Knudsen number equal to 0.3 (slip regime $^{8}$ ). Finally the results obtained for these gases were compared together and compared also with those previously obtained with helium up to $K n=25$, with $\mathcal{P}=3$.

\section{A. Hydrodynamic and slip regime}

The experimental dimensionless mass flow rate data were fitted with a second order polynomial form:

$$
S_{f}^{\exp }=1+A^{\exp } K n_{m}+B^{\exp } K n_{m}^{2}
$$

by using a nonlinear least squares method, as detailed in Ref. 3 , in order to describe the slip regime. The coefficients, $A^{\exp }$ and $B^{\exp }$, obtained by applying the nonlinear least squares Marquard-Levenberg algorithm to the measured values of the mass flow rate, normalized according to Eq. (6), are reported in Table II. The uncertainty on these coefficients was estimated using standard deviation. From the comparison of the theoretical [Eq. (6)] and experimental [Eq. (11)] mass flow rate expressions, coefficient $A_{1}$ may be related to the experimental coefficient: 
TABLE III. The most pertinent slip coefficients and accommodation coefficients are in bold characters. The helium results are derived from Ref. 5. The wall material is silicon.

\begin{tabular}{lccccc}
\hline \hline Gas & $\begin{array}{c}\text { Molar mass } \\
(\mathrm{g} / \mathrm{mol})\end{array}$ & $\sigma_{p}^{1 \mathrm{st}}$ & $\sigma_{p}^{2 \text { nd }}$ & $\alpha^{1 \mathrm{st}}$ & $\alpha^{2 \text { nd }}$ \\
\hline $\mathrm{He}$ & 4.00 & $\mathbf{1 . 1 5 3} \pm \mathbf{0 . 0 0 8}$ & $\ldots$ & $\mathbf{0 . 9 3 3} \pm \mathbf{0 . 0 0 3}$ & $\ldots$ \\
$\mathrm{N}_{2}$ & 28.02 & $1.253 \pm 0.011$ & $\mathbf{1 . 1 0 4} \pm \mathbf{0 . 0 1 0}$ & $0.889 \pm 0.004$ & $\mathbf{0 . 9 5 6 \pm 0 . 0 0 5}$ \\
$\mathrm{Ar}$ & 39.95 & $1.355 \pm 0.022$ & $\mathbf{1 . 2 0 5} \pm \mathbf{0 . 0 6 4}$ & $0.848 \pm 0.008$ & $\mathbf{0 . 9 1 0 \pm 0 . 0 2 8}$ \\
\hline \hline
\end{tabular}

$$
A_{1}=\frac{\sigma_{p}}{k_{\lambda}}=\frac{A^{\exp }}{6}, \quad A_{2}=\frac{B^{\exp }}{6 \ln \mathcal{P}} \frac{(\mathcal{P}-1)}{(\mathcal{P}+1)} .
$$

The previous relation allows the experimental estimations of the velocity slip coefficient $\sigma_{p}$ given in Table III. The values so obtained for $\sigma_{p}$ are slightly different from two theoretical predictions of these slip coefficient: $\sigma_{p}=1.012$ given by Ref. 21 and $\sigma_{p}=1.016$ given by Ref. 13. Both these theoretical values have been obtained from the kinetic equation BGK model under assumption of a full accommodation of the molecules at the wall. The differences between our experimental results and the two last theoretical results confirmed that the silicon cannot be considered like a full diffuse surface. Consequently the knowledge of the TMAC appeared important, and we calculated it to improve the understanding of the gas/surface interaction.

Thus, we derived an experimental value of the accommodation coefficient using the Maxwell diffuse-scattering model. We compared our mass flow rate measurements to the theoretical results of Loyalka which led to deduce the accommodation coefficient as proposed in Ref. 19. In this work, ${ }^{19}$ the authors calculated the values of the slip coefficient from the BGK kinetic model for various values of the accommodation coefficient using the Maxwell scattering kernel as kinetic boundary condition. A simple expression associating the slip coefficient and the accommodation coefficient was also derived using a simple interpolation in Ref. 19 and improved in Ref. 22. In so doing, the Knudsen layer effects are taken into account through the slip coefficient ${ }^{22}$

$$
\sigma_{p}(\alpha)=\frac{2-\alpha}{\alpha}\left[\sigma_{p}(1)-0.1211(1-\alpha)\right],
$$

where $\sigma_{p}(1)$ the slip coefficient for $\alpha=1$ equals 1.016 , i.e., the value obtained by Ref. 13 taking into account the influence of the Knudsen layer. Only this last definition of the slip coefficient, more precise than the well known Maxwell expression, ${ }^{11}$ will be used in this article. Moreover, some remarks can be done.

For $\mathrm{N}_{2}$ and Ar the second order treatment appeared us the most pertinent in slip regime using the same criteria as those used in Ref. 5. The values of the main parameters used as criteria appear in Table II. On the [0-0.3] Knudsen range we calculated, notably, the determination coefficient $r^{2}$, the squared residual sum $s_{r}=\sqrt{(1 / n-p) \sum_{i=1}^{n} e_{i}^{2}}$, and the standard error on the fit curves $E_{s}=\sqrt{(1 / n) \sum_{i=1}^{n} e_{i}^{2}} / \overline{S^{\exp }}$. Here $e_{i}=S_{i}^{\exp }$ $-S_{f_{i}}^{\exp }$ is the local difference between the measured and fitting values and it represents the local fitting error, $n$ is the number of measurements, $p$ is the number of the unknown coeffi- cients of the fitting model, and $\overline{S^{\exp }}=(1 / n) \sum S_{i}^{\exp }$ is the averaged value of the measured values. Analyzing the values of these coefficients given in Table II, respectively, for first and second order approaches, we found that the determination coefficient of second order is closer to one than that of first order. Moreover the squared residual sum and the standard error are smaller for the second order fitting. As well known we may thus estimate that the second order fitting is of better quality. Furthermore, as detailed in Ref. 5, this analysis was confirmed by studying the form of the distribution of residuals $e_{i}$; the second order parameters appeared equidistributed, contrarily to that of first order. Thus we retained for these gases the second order values (see Tables II and III).

The different values of $\alpha$ obtained for different gases revealed the accommodation coefficient tendency to increase when the molecular mass decreases. Consequently, the first slip coefficients are then decreasing with the mass. We already tested similar gas/wall interactions in the slip regime through a microtube ${ }^{8}$ and we found the same tendency.

In addition, in a previous work we considered helium in plane microchannel where the second order is not significant and not sufficiently important to be measured with a suitable precision. ${ }^{5}$ Thus to compare in a consistent manner the $\alpha$ variations according to the molecular mass we referred to the first order for all the gases given in Tables II and III: in this frame the increase in $\alpha$ with the decreasing mass is confirmed when including helium (see Tables II and III).

Moreover in Ref. 5 studying the helium flow slip regime, in a plane microchannel, we obtained a nonsignificant second order effect for this gas. Then, we could not extract a meaningful second order term, due to a relative error on this second order term. Therefore, in order to compare the trend of $\alpha$ following the molecular mass using the same order for all the gases, we referred only to the first order results; then the increase in $\alpha$ with a decreasing mass is observed for all the gases analyzed in this work (see Tables II and III).

\section{First order}

Naturally, the small number of works and the small number of studied gases do not yet allow us to conclude in favor of an absolute decrease in the accommodation coefficient with the increasing in the molecular mass. Other parameters could reveal their influence leading us to moderate our conclusion. Nevertheless when comparing our results obtained in microchannel to other results given in the literature for the same geometry, more especially to Refs. 3 and 2, it is clear that the same tendency was generally found. 
TABLE IV. Second order effects: comparison in tube $[D=25.2 \mu \mathrm{m}$, subscript $\left.(\cdots)_{t}\right]$ and plane microchannel $\left[H=9.38 \mu \mathrm{m}\right.$, subscript $\left.(\cdots)_{\mathrm{ch}}\right]$.

\begin{tabular}{lcccc}
\hline \hline Gas & $\left(B^{\exp } / A^{\exp }\right)_{\mathrm{ch}}$ & $\left(B^{\exp } / A^{\exp }\right)_{t}$ & $\left(\sigma_{2 p} / \sigma_{p}\right)_{\mathrm{ch}}$ & $\left(\sigma_{2 p} / \sigma_{p}\right)_{t}$ \\
\hline $\mathrm{He}$ & 0.059 & 0.847 & 0.024 & 0.138 \\
$\mathrm{~N}_{2}$ & 0.700 & 1.429 & 0.298 & 0.217 \\
$\mathrm{Ar}$ & 0.585 & 1.836 & 0.249 & 0.260 \\
\hline \hline
\end{tabular}

A first interpretation of the observed tendency could be that the wall/gas interaction tends to be totally diffuse with decreasing molecule mass, because of both dynamical and structural reasons. First, when impinging the wall, the molecules forget all the more easily their incoming direction as their momentum is smaller. Second, the wall asperities can manage and scatter more easily the incoming molecules when their weight becomes smaller. This second aspect is enhanced when comparing only monoatoming molecules because then a decreasing weight means also a decreasing size.

\section{Second order}

Furthermore we observed that the second order effect and the second slip coefficient [Eqs. (5) and (6)] also decrease with decreasing molecular mass (see Table II). Let us note that this property may again be extended to He, considering that its second order effect is negligible. ${ }^{5}$ Again we can note that in a previous work, in a microtube, ${ }^{8}$ the same tendency was observed regarding the molecular mass dependence of the second order coefficient. Let us note that the material of the microtubes used in Ref. 8 is fused silica. The wall roughness for the microtube was estimated by the same MEB technique and found less than $20 \mathrm{~nm}$.

Finally, comparing the results obtained in Ref. 8 with the present results, we can remark that the second order effect observed in microtubes for the same gases was more important than the present corresponding effect observed in plane microchannels. More precisely, when considering the second order coefficient $B^{\text {th }}$ or $B^{\exp }$ involved in the mass flow rate expressions (7) and (11), we can observe this tendency whatever the gas; but regarding the second order slip coefficient $\sigma_{2 p}$ (or the $\sigma_{2 p} / \sigma_{p}$ ratio) this hierarchy tube/channel is maintained if we restrict the comparison to the monoatomic gases (see Table IV).

\section{B. Transitional and free molecular regime}

The measured mass flow rates are given in Fig. 2 in a nondimensional form according to Eq. (10) for three gases (He, $\mathrm{N}_{2}$, and Ar). The results were plotted as a function of the rarefaction parameter $\delta_{m}$ associated with the hard sphere model.

In order to compare this measured mass flow rate with the available theoretical results, we used, as previously said, the numerical solutions of the BGK model equation; especially those of Ref. 19 describing two parallel plates and using variable accommodation coefficient and those ${ }^{17}$ describing rectangular channels and using diffuse boundary

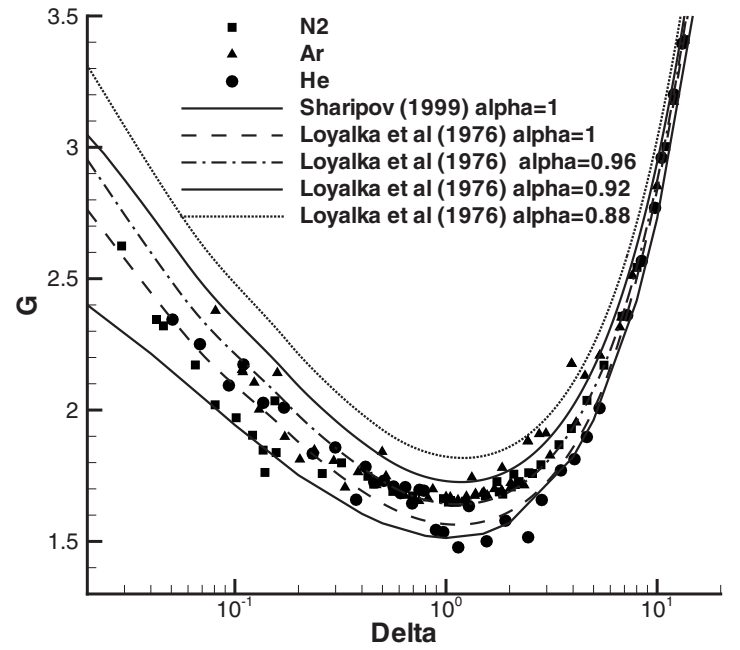

FIG. 2. Experimental mass flow rate in the channel, calculated according to Eq. (10) and theoretical curves (Ref. 17): (full accommodation) rectangular channel $H / w=0.02$ or (Ref. 19) (parallel plates) with $\alpha=0.88,0.92,0.96$, and 1.0. The magnitude of experimental uncertainty is smaller than $4.5 \%$.

conditions on the walls. As mentioned in a previous section, we integrated the nondimensional quantity $Q$ [Eq. (9)] over $\delta$ in order to compare the results with our measurements where the pressure varies essentially along the channel. In Fig. 2, the theoretical mass flow rates obtained after integration were represented. Various theoretical curves for various coefficients $\alpha$ deduced from the approach of Ref. 19 were drawn. From visual graphic comparison of our results with Loyalka's curves, it seems that for helium the most convenient value of $\alpha$ is close to 1.0, those of nitrogen are rather close to 0.96 , and for argon close to 0.94 . These results confirm that a full accommodation on the surface is generally inappropriate in the transitional range as well as in slip regime. Moreover, it seems that, when the same theoretical frame is applied to analyze experimental results, the same accommodation coefficient (of tangential momentum) may be accepted in slip as in transitional regimes.

From the curve trends in Fig. 2, we can directly deduce some comments. First, the accommodation coefficient and the reduced mass flow rate have the same trend according to the molecular mass of the gases as those noticed in slip regime (presently in microchannel and previously in microtube ${ }^{8}$ ). Then, in all the cases observed here, this reveals the same trend with respect to mass: $\alpha$ decreases when the molecular mass increases. This tendency is confirmed and generalized in a recent survey ${ }^{23}$ where a quoted article ${ }^{24}$ presents results obtained from another experimental technique in good agreement with our values: thus the ratios between the accommodation coefficient of various gases are very close (the ratios $\alpha_{\mathrm{Ar}} / \alpha_{\mathrm{He}}$ are, respectively, 0.93 in Ref. 24 and 0.95 in the present work; note that in Ref. 24 the absolute values have not been measured).

Conversely, to our knowledge, only one group of researchers ${ }^{25-27}$ found sometimes a different tendency about the accommodation dependence on the molecular mass, but in very different conditions. Especially in Ref. 26 when us- 
ing a different geometry (in cylindrical microtube), for very low pressures (corresponding here to a free molecular regime) and for a clean surface (i.e., with solid surfaces treated in order to not suffer the contamination from the gas adsorption) these authors found another TMAC trend for the various gases according to their molecular mass. Various reasons could explain this surprising behavior. We will not analyze here all the possibilities. But, the main particular feature of these authors' experiments is the state of clean surface characterizing their wall surfaces while all the other authors previously quoted considered in their experiments, like we did ourselves, rather the case of surface in contact with the various flowing gases in normal and permanent conditions. In other words this is the case of surface suffering probably an important contamination from the flowing gas, and in these conditions the nature of the surface material does not significantly influence the accommodation process, at least for monoatomic gases. ${ }^{23}$ Thus the differences in the result could be explained considering that in Ref. 26 the gases could have a different behavior from a chemical point of view (wall atom/gas molecule interaction, adsorption). Moreover, we can recall the remark made in Sec. IV A 1 and underlined in the Conclusion: we do not exclude that other parameters influence this result and, in some cases, could change the tendency.

Looking at Fig. 2 from the slip regime toward the free molecular regime $\left(\delta_{m} \rightarrow 0\right)$, the influence of the wall appeared first through the accommodation coefficient, so the departure between the experimental points and both theoretical diffuse curves increases for $\delta_{m}$ close to 1 . In this zone, although describing a rectangular channel, the results obtained from Ref. 17 do not agree very well with our results, because based on a complete diffusive reflection, the flow is not enough rarefied (and the gas-gas collisions not enough vanishing) to allow the collisions on the small lateral walls to counterbalance the influence of the incomplete diffusive accommodation. Only after (for $\delta_{m}$ near to 0.1 ) the rarefaction influence acts through the geometry of the section, the measurements appear then "attracted" toward the curve from Ref. 17; for some gases, they become then closer to the finite section model ${ }^{17}$ than to the infinite section model corresponding to the "good" experimental accommodation coefficient quoted above. ${ }^{16}$

Globally the comparison theory/experiments appears satisfactory if considering the experimental error. Even if one can observe (see Fig. 2), for a few experimental points, a departure from the theoretical value slightly overcoming the experimental error. Two explanations may be given:

- A reason may be found in the comment at the end of Sec. III: in some conditions we cannot exclude a modeling error overcoming the experimental error.

- For the points located at very low $\delta$ we do not have a completely suitable theoretical model. As previously said, in this case the finite width section of the channel plays an important role.

Therefore, for a more accurate analysis of the combined effects of the partial accommodation and of the limited rectan- gular section of the channel, it would have been interesting to compare the measurements with theoretical models taking into account both of these effects. However, this kind of theoretical results could not be found in the literature.

\section{CONCLUSIONS}

Mass flow rate measurements in a microchannel were carried out for three different gases under isothermal steady state conditions. A wide Knudsen number range, from the continuum slip regime to the near free molecular regime, was explored. In the slip regime, a continuum approach (Navier-Stokes equations) involving general formulations of first or second order slip boundary conditions (depending on the gas) were used in order to obtain the experimental velocity slip and the accommodation coefficients associated with the Maxwell kinetic boundary condition. In the transitional and near free molecular regimes the measured values of the mass flow rate were compared to the corresponding values given by the kinetic approaches and the accommodation coefficient values were deduced again. These last values were found between 0.94 and 1.0, i.e., slightly higher than those obtained in slip regime from the continuum approach, but they behave similarly as a function of molecular mass; when using a same approach for all the gases studied in the present work, the values of the accommodation coefficients of the tangential moment increase when the molecular mass decreases whatever the regime. Correlatively, the first and second slip coefficients decrease with the molecular mass.

But as stated in Sec. IV A 1 we do not conclude here that $\alpha$ is decreasing with increasing molecular mass whatever the conditions (whatever the wall material, whatever the roughness or even whatever the gas). We only sustained that for the gases studied in the conditions of this work this tendency is verified. We agree to the idea that other parameters (the viscosity, the internal structure or more basically the characteristic length of the interaction potential) could moderate and modulate this tendency, depending on the gas and such influences could appear in the frame of a larger study.

Finally in the slip regime, we noticed that the second order appears generally more important when using the cylindrical microtube than in plane microchannel. In this study the wall materials of the tube and channel are, respectively, different. But other results ${ }^{28,29}$ obtained in various tubes of fused silica seem to confirm these results. In the future we plan to show that the second order effect continuously increases with the wall curvature and then to model this feature.

\section{ACKNOWLEDGMENTS}

We would like to acknowledge T. Ewart for his help in the experimental data preparation. The authors are grateful to the Conseil Régional Provence Alpes Côtes d'Azur its financial support. The research leading to these results received funding from the European Community's Seventh Framework Programme (ITN-FP7/2007-2013) under Grant No. N 215504. 
${ }^{1}$ S. Colin, P. Lalonde, and R. Caen, "Validation of a second-order slip flow model in a rectangular microchannel," Heat Transf Eng. 25, 23 (2004).

${ }^{2}$ E. B. Arkilic, K. S. Breuer, and M. A. Schmidt, "Mass flow and tangential momentum accomodation in silicon micromachined channels," J. Fluid Mech. 437, 29 (2001).

J. Maurer, P. Tabeling, P. Joseph, and H. Willaime, "Second-order slip laws in microchannels for helium and nitrogen," Phys. Fluids 15, 2613 (2003).

${ }^{4}$ T. Ewart, P. Perrier, I. A. Graur, and J. G. Méolans, "Mass flow rate measurements in gas micro flows," Exp. Fluids 41, 487 (2006).

${ }^{5}$ T. Ewart, P. Perrier, I. A. Graur, and J. G. Méolans, "Mass flow rate measurements in microchannel, from hydrodynamic to near free molecular regimes," J. Fluid Mech. 584, 337 (2007).

${ }^{6}$ G. Karniadakis and A. Beskok, Microflows: Fundamentals and Simulation (Springer, New York, 2002).

${ }^{7}$ Y. Sone, Kinetic Theory and Fluid Mechanics (Birkhäuser, Boston, 2002).

${ }^{8}$ T. Ewart, P. Perrier, I. A. Graur, and J. G. Méolans, "Tangential momentum accomodation in microtube," Microfluid. Nanofluid. 3, 689 (2007).

${ }^{9}$ S. Chapman and T. G. Cowling, The Mathematical Theory of NonUniform Gases (Cambridge University Press, Cambridge, 1952).

${ }^{10}$ C. Cercignani, Mathematical Methods in Kinetic Theory, 2nd Ed. (Plenum, New York, 1990).

${ }^{11}$ J. C. Maxwell, "On stress in rarefied gases arising from inequalities of temperature," Philos. Trans. R. Soc. London, Ser. B 170, 231 (1878).

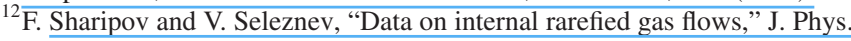
Chem. Ref. Data 27, 657 (1998).

${ }^{13}$ C. Cercignani and A. Daneri, "Flow of a rarefied gas between two parallel plates," J. Appl. Phys. 34, 3509 (1963).

${ }^{14}$ S. K. Loyalka, "Kinetic theory of thermal transpiration and mechanocaloric effects II," J. Chem. Phys. 63, 4054 (1975).

${ }^{15}$ T. Ohwada, Y. Sone, and K. Aoki, "Numerical analysis of the Poiseuille and thermal transpiration flows between two parallel plates on the basis of the Boltzmann equation for hard sphere molecules," Phys. Fluids A 1, 2042 (1989).

${ }^{16}$ S. K. Loyalka, T. S. Stvorik, and H. S. Park, "Poiseulle flow and thermal creep flow in long, rectangular channels in the molecular and transition flow regimes," J. Vac. Sci. Technol. 13, 1188 (1976).
${ }^{17}$ F. Sharipov, "Rarefied gas flow through a long rectangular channel," J. Vac. Sci. Technol. A 17, 3062 (1999).

${ }^{18}$ F. Sharipov, "Non-isothermal gas flow through rectangular microchannels," J. Micromech. Microeng. 9, 394 (1999).

${ }^{19}$ S. K. Loyalka, N. Petrellis, and S. T. Stvorick, "Some numerical results for the bgk model: Thermal creep and viscous slip problems with arbitrary accommodation of the surface," Phys. Fluids 18, 1094 (1975).

${ }^{20}$ I. A. Graur, J. G. Méolans, and D. E. Zeitoun, "Analytical and numerical description for isothermal gas flows in microchannels," Microfluid. Nanofluid. 2, 64 (2006).

${ }^{21}$ M. N. Kogan, Rarefied Gas Dynamics (Plenum, New York, 1969).

${ }^{22} \mathrm{~F}$. Sharipov, "Data on the velocity slip and temperature jump coefficients," in Thermal and Mechanical Simulation and Experiments in MicroElectronics and Micro-Systems, Proc. 5th Int. Conf. EuroSimE, edited by L. J. Ernst, G. Q. Zhang, P. Rodgers, and O. de Saint Leger (Shaker, Belgium, 2004), pp. 243-249.

${ }^{23}$ A. Agrawal and S. V. Prabhu, "Survey on measurement of tangential momentum accommodation coefficient," J. Vac. Sci. Technol. A 26, 634 (2008).

${ }^{24}$ T. Gronych, R. Ulman, L. Peksa, and P. Repa, "Measurements of the relative momentum accommodation coefficient for different gases with a viscosity vacuum gauge," Vacuum 73, 275 (2004).

${ }^{25}$ P. E. Suetin, B. T. Porodnov, V. G. Chernayk, and S. F. Borisov, "Poiseuille flow at arbitrary Knudsen number and tangential momentum accommodation,” J. Fluid Mech. 60, 581 (1973).

${ }^{26}$ O. V. Sazhin, S. F. Borisov, and F. Sharipov, "Accommodation coefficient of tangential momentum on atomically clean and contaminated surfaces," J. Vac. Sci. Technol. A 19, 2499 (2001).

${ }^{27}$ B. T. Porodnov, P. E. Suetin, S. F. Borisov, and V. D. Akinshin, "Experimental investigation of rarefied gas flow in different channels," J. Fluid Mech. 64, 417 (1974).

${ }^{28}$ T. Ewart, "Etude des écoulements gazeux isothermes en microconduits: Du régime hydrodynamique au proche régime molćulaire libre," Ph.D. thesis, Provence Ubiversity, 2007 (in French).

${ }^{29}$ P. Perrier, "Instrumentation et physisique expérimentale appliquées à la spectrométrie de masse et aux écoulemets gazeux," Habilitating Thesis, Provence University, 2008 (in French). 